Kyushu J. Math.

Vol. 56, 2002, pp. 123-135

\title{
CANCELLATION LAW FOR INFINITE COMPLEXES
}

\author{
Yoshimi SHITANDA \\ (Received 28 August 2000 and revised 17 November 2000)
}

\section{Introduction}

In this paper we work in the category of based $C W$-complexes and based maps. We are able to construct a homotopy category as usual. In the homotopy category, there exist wedge sums $\vee$ and product operations $\times$. We propose the following problems. We use the same terminologies and notation of [6] in this paper.

Problem 1. Is a decomposition into wedge sums uniquely determined? That is, if $X_{1} \vee X_{2} \vee \cdots \vee X_{n}$ and $Y_{1} \vee Y_{2} \vee \cdots \vee Y_{n}$ are homotopy equivalent and $X_{i}$ and $Y_{i}$ are prime spaces, then does it hold that $X_{i}$ and $X_{\sigma(i)}$ are homotopy equivalent for a permutation $\sigma$ ?

Problem 2. Does a cancellation law hold in the homotopy category? That is, if $X_{1} \vee X_{2} \vee \cdots \vee X_{n}$ and $Y_{1} \vee Y_{2} \vee \cdots \vee Y_{n}$ are homotopy equivalent and also for $X_{1}$ and $Y_{1}$, then does it hold that $X_{2} \vee \cdots \vee X_{n}$ and $Y_{2} \vee \cdots \vee Y_{n}$ are homotopy equivalent?

Generally the answers to these problems are no. For the homotopy category of finite $C W$-complexes, Freyd [1] and other topologists $[\mathbf{2 - 4 , 8}$ ] gave some examples and studied them in detail. For the homotopy category of infinite $C W$-complexes, the author [6] gave some examples and studied the above problems. In this paper, we continue their study and obtain a solution. In this paper, we assume that a phantom map is a map from $\Sigma^{i} L(I, J)$ to $S^{i+3}$ for $i \geq 0$ where $L(I, J)$ is a space obtained by mixing localized spaces $C P_{I}^{\infty}$ and $\Omega\left(S^{3}\right)_{J}$ over $K(Q, 2)$. Our main theorems are as follows.

THEOREM 1.1. Let $f$ be a phantom map of infinite order and $f_{i}=a_{i} f$ for integers $a_{i},(i=1,2, \ldots, n)$. Then, there exist phantom maps $g_{i}(i=1,2, \ldots, n)$ of infinite order satisfying the following conditions:

(1) $\bigvee_{i=1}^{n} C\left(f_{i}\right)$ and $\bigvee_{i=1}^{n} C\left(g_{i}\right)$ are homotopy equivalent; 
(2) $g_{i}=b_{i} g$ for a phantom map $g$ and integers $b_{i}(i=1,2, \ldots, n)$ and $b_{i+1}$ is divided by $b_{i}$ for $i=1,2, \ldots, n-1$.

THEOREM 1.2. Let $f_{i}$ and $g_{i}(i=1,2, \ldots, n)$ be phantom maps of infinite order and $f_{1}$ and $g_{1}$ homotopic maps. If two wedge sums $\bigvee_{i=1}^{n} C\left(f_{i}\right)$ and $\bigvee_{i=1}^{n} C\left(g_{i}\right)$ are homotopy equivalent, $\bigvee_{j=2}^{n} C\left(f_{j}\right)$ and $\bigvee_{j=2}^{n} C\left(g_{j}\right)$ are homotopy equivalent.

THEOREM 1.3. Let $f_{i}(i=1,2, \ldots, n)$ be phantom maps of finite order. Then there exist phantom maps $g_{i}(i=1,2, \ldots, n)$ of finite order satisfying the following conditions:

(1) $\bigvee_{i=1}^{n} C\left(f_{i}\right)$ and $\bigvee_{i=1}^{n} C\left(g_{i}\right)$ are homotopy equivalent;

(2) $\operatorname{ord}\left(g_{i}\right)$ is divided by $\operatorname{ord}\left(g_{i+1}\right)$.

THEOREM 1.4. Let $f_{i}$ and $g_{i}(i=1,2, \ldots, n)$ be phantom maps of finite order such that $\operatorname{ord}\left(f_{i}\right)$ is divided by ord $\left(f_{i+1}\right)$ and also for the $g_{i}$. If $\bigvee_{i=1}^{n} C\left(f_{i}\right)$ and $\bigvee_{i=1}^{n} C\left(g_{i}\right)$ are homotopy equivalent, it holds that $\operatorname{ord}\left(f_{i}\right)=\operatorname{ord}\left(g_{i}\right)$ for $i=1,2, \ldots, n$.

\section{Preliminary}

We define a space $L(I, J)$ in which to study our problems. Let $C P^{\infty}$ and $\Omega\left(S^{3}\right)$ be an infinite-dimensional projective space and a loop space of a 3 -sphere, respectively, and spaces $C P_{I}^{\infty}$ and $\Omega\left(S^{3}\right)_{J}$ their localizations at sets $I$ and $J$ of prime numbers. Let $L(I, J)$ be a space given by the following pull-back diagram where $(I, J)$ is a partition of all primes, $I$ a non-empty set and the maps from $\Omega\left(S^{3}\right)_{J}$ and $C P_{I}^{\infty}$ to $K(Q, 2)$ are rationalizations:

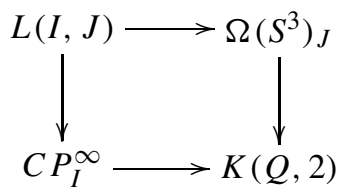

The homology groups of the space $L(I, J)$ are a free group $Z$ in each even dimension and zero in each odd dimension. Note that the space $L(I, J)$ is an $\mathrm{H}$ space. Although the calculation of a homotopy set $\left[\Sigma^{i} L(I, J), S^{i+3}\right]$ is difficult, it is comparatively easy to calculate the homotopy set $P h\left(\Sigma^{i} L(I, J), S^{i+3}\right)$ of phantom maps. Now we state our results of $[6,7]$.

TheOREM 1. $P h\left(\Sigma^{i} L(I, J), S^{i+3}\right)$ is isomorphic to $\hat{Z}_{I} / Z_{I} \oplus \bigoplus_{q \in J} Z_{q} \infty$ where $\hat{Z}_{I} / Z_{I}$ is a vector space over $Q$ with uncountable basis and $Z_{q} \infty$ is a torsion group of a component of prime $q$ of $Q / Z$. 
In the previous papers $[\mathbf{5}, \mathbf{6}]$, we classified the homotopy type of mapping cones $C(f)$ of phantom map $f: \Sigma^{i} L(I, J) \rightarrow S^{i+3}$.

THEOREM 2. Let $f$ and $g$ be phantom maps from $\Sigma^{i} L(I, J)$ to $S^{i+3}$. Then the loop spaces of the mapping cones $\Omega^{i} C(f)$ and $\Omega^{i} C(g)$ are homotopy equivalent if and only if $f$ and $\pm g$ are homotopic for $i=0,1, \ldots, \infty$.

By making use of these theorems, we studied cancellation and non-cancellation phenomena under the wedge sum operation in the homotopy category. We obtained the partial results for the phenomena in a previous paper [6]. In this paper, we prove some results about the wedge sum decomposition similar to the fundamental theorem of finitely generated abelian groups. From now on, by phantom maps we mean maps from $\Sigma^{i} L(I, J)$ to $S^{i+3}$ for $i \geq 0$.

THEOREM 3. Let $f_{i}$ and $f_{i}^{\prime}(i=1,2, \ldots, m)$ be phantom maps of infinite order and $g_{j}$ and $g_{j}^{\prime}(i=1,2, \ldots, n)$ phantom maps of finite order. If two wedge sums $\bigvee_{i=1}^{m} C\left(f_{i}\right) \vee \bigvee_{j=1}^{n} C\left(g_{j}\right)$ and $\bigvee_{i=1}^{m} C\left(f_{i}^{\prime}\right) \vee \bigvee_{j=1}^{n} C\left(g_{j}^{\prime}\right)$ are homotopy equivalent, $\bigvee_{i=1}^{m} C\left(f_{i}\right)$ and $\bigvee_{i=1}^{m} C\left(f_{i}^{\prime}\right)$ are homotopy equivalent and also for $\bigvee_{j=1}^{n} C\left(g_{j}\right)$ and $\bigvee_{j=1}^{n} C\left(g_{j}^{\prime}\right)$

Proof. Set $F=\operatorname{Diag}\left(f_{1}, \ldots, f_{m}\right)$ and $F \oplus G=\operatorname{Diag}\left(f_{1}, \ldots, f_{m}, g_{1}, \ldots, g_{n}\right)$ where the right-hand sides are diagonal matrices with components $f_{1}, \ldots, g_{1}, \ldots$ etc, and also for $F^{\prime}, F^{\prime} \oplus G^{\prime}$. A map $\phi$ is a homotopy equivalence between $C(F \oplus G)$ and $C\left(F^{\prime} \oplus G^{\prime}\right)$.

Consider the following diagram:

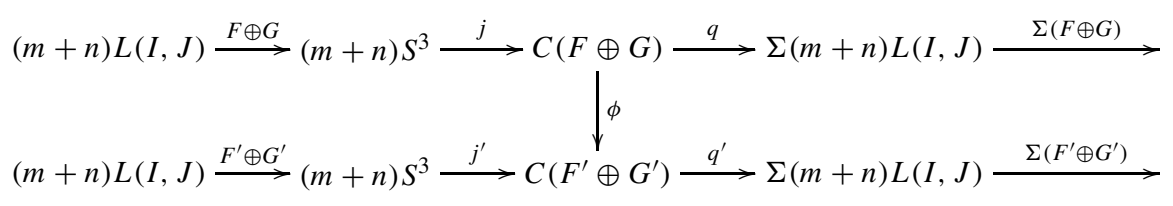

where the horizontal sequences are Puppe sequences induced by $F \oplus G:$ : $m+$ $n) L(I, J) \rightarrow(m+n) S^{3}$ and $F^{\prime} \oplus G^{\prime}:(m+n) L(I, J) \rightarrow(m+n) S^{3}$, respectively. We will find a map from $(m+n) S^{3}$ to $(m+n) S^{3}$ which makes the diagram commutative. A homotopy equivalence $\phi: C(F \oplus G) \rightarrow C\left(F^{\prime} \oplus G^{\prime}\right)$ gives the following matrix which is a homomorphism on three-dimensional homology groups:

$$
\begin{aligned}
H_{3}(\phi) & =\left(A_{i j}\right) \quad(i, j=1,2,3,4) \\
A_{i j}: H_{3}\left(X_{j}\right) & \rightarrow H_{3}\left(X_{i}\right)
\end{aligned}
$$


where $X_{i}$ is $m S^{3}, n S^{3}, m \Sigma L(I, J)$ and $n \Sigma L(I, J)$ for $i=1,2,3$ and 4, respectively. Small matrices $A_{31}$ and $A_{41}$ of the matrix are zero by the same method of Theorem 2.3 and Proposition 3.2 in [6], and small matrices $A_{13}, A_{14}, A_{23}$ and $A_{24}$ are zero by (co)homology. Hence, we have obtained the following matrix:

$$
\left(\begin{array}{cccc}
A_{11} & A_{12} & 0 & 0 \\
A_{21} & A_{22} & 0 & 0 \\
0 & A_{32} & A_{33} & A_{34} \\
0 & A_{42} & A_{43} & A_{44}
\end{array}\right) .
$$

Consider the following composition:

$$
(m+n) L(I, J) \stackrel{F \oplus G}{\longrightarrow}(m+n) S^{3} \stackrel{j}{\longrightarrow} C(F \oplus G) \stackrel{\phi}{\longrightarrow} C\left(F^{\prime} \oplus G^{\prime}\right) \stackrel{r}{\longrightarrow} n S^{3}
$$

where the first three terms are a Puppe sequence of a map $F \oplus G$ and the last map $C\left(F^{\prime} \oplus G^{\prime}\right) \rightarrow n S^{3}$ is given by Proposition 3.4 in [6]. Since the composed map is homotopic to a constant map, we can easily see $A_{21}=0$ by Proposition 3.2 in [6]. In the same way as above, we can see that the $i$ th column of $A_{32}, A_{42}$ is a multiple of $\operatorname{ord}\left(g_{i}\right)(1,2, \ldots, n)$. By using Propositions 3.4 and 3.5 in [6], we can also construct a map $\psi: C(F \oplus G) \rightarrow C(F \oplus G)$ which induces the following matrix on $H_{3}(C(F \oplus G))$ :

$$
\left(\begin{array}{cccc}
I & 0 & 0 & 0 \\
0 & I & 0 & 0 \\
0 & E_{1} & I & 0 \\
0 & E_{2} & 0 & I
\end{array}\right)
$$

By choosing $E_{1}$ and $E_{2}$ well, $H_{3}(\phi \psi)$ becomes a matrix with $A_{32}=0, A_{42}=0$. Hence we may assume that a matrix $A$ has the following form:

$$
\left(\begin{array}{cccc}
A_{11} & A_{12} & 0 & 0 \\
0 & A_{22} & 0 & 0 \\
0 & 0 & A_{33} & A_{34} \\
0 & 0 & A_{43} & A_{44}
\end{array}\right) .
$$

From the above matrix, we obtain a map $\alpha:(m+n) S^{3} \rightarrow(m+n) S^{3}$ and the following commutative diagram:

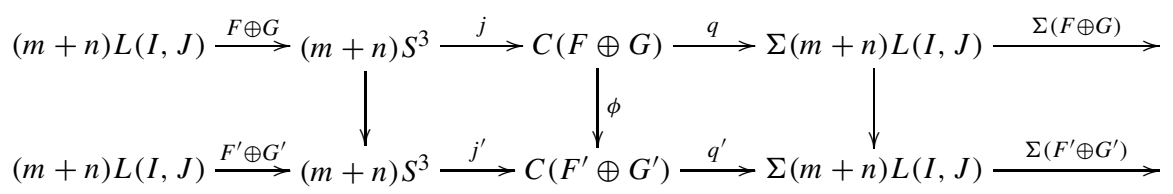


Hence we obtain the following equation from the last square:

$$
\left(\begin{array}{cc}
A_{11} & A_{12} \\
0 & A_{22}
\end{array}\right)\left(\begin{array}{cc}
F & 0 \\
0 & G
\end{array}\right)=\left(\begin{array}{cc}
F^{\prime} & 0 \\
0 & G^{\prime}
\end{array}\right)\left(\begin{array}{ll}
A_{33} & A_{34} \\
A_{43} & A_{44}
\end{array}\right)
$$

From this equation, we have $A_{12} G=F^{\prime} A_{34}$. Since the components of $G$ are of finite order, the components of the left-hand side are of finite order. Since the components of the right-hand side are of infinite order or zero, we see that $A_{34}=0$. From the result, we obtain $A_{11} F=F^{\prime} A_{33}$ and $A_{22} G=G^{\prime} A_{44}$ where $A_{11}, A_{22}, A_{33}$ and $A_{44}$ are unimodular matrices. Hence we obtain the result.

The following theorem is proved in a way entirely similar to the above theorem and Theorem 2.1 of [6].

THEOREM 4. Let $f_{i}(i=1,2, \ldots, m)$ be phantom maps of infinite order which are linearly independent over $Q$ and also for $g_{i}(i=1,2, \ldots, m)$. Let $f_{i j}=c_{i j} f_{i}$ $\left(j=1, \ldots, p_{i}\right), g_{i j}=d_{i j} g_{i}\left(j=1,2, \ldots, q_{i}\right)$ where $c_{i j}$ and $d_{i j}$ are non-zero rational numbers. If two wedge sums $\bigvee_{i, j} C\left(f_{i j}\right)$ and $\bigvee_{i, j} C\left(g_{i j}\right)$ are homotopy equivalent, $\bigvee_{j=1}^{p_{i}} C\left(f_{i j}\right)$ and $\bigvee_{j=1}^{q_{k}} C\left(g_{k j}\right)$ are homotopy equivalent where $k=\sigma(i)$ for a permutation $\sigma$ and multiples of $f_{i}$ and $g_{\sigma(i)}$ are homotopic maps for each $i$.

\section{Main theorems}

Let $f$ be a phantom map of infinite order. Then, the following formula is given in section 2 in [6] for suspended maps $f$ and $g$ :

$$
C(\text { maf }) \vee C(n a f) \simeq C(\text { mnaf }) \vee C(a f)
$$

where $m$ and $n$ are relatively prime integers and $a$ is an integer. It is proved by the following matrix calculation in the above case that

$$
\left(\begin{array}{cc}
m f & 0 \\
0 & n f
\end{array}\right)\left(\begin{array}{cc}
1 & -n t \\
1 & m s
\end{array}\right)=\left(\begin{array}{cc}
m & -t \\
n & s
\end{array}\right)\left(\begin{array}{cc}
f & 0 \\
0 & m n f
\end{array}\right)
$$

where $m s+n t=1$. Hence the uniqueness of decomposition fails by the formula (3.1). From the formula, we have a normal form of the decomposition which is a unique decomposition in a sense. At first we prove the formula (3.1) for the general case, that is, non-suspended maps $f, g$.

Now we have to prepare a lemma to construct a homotopy equivalence for nonsuspended spaces. 
LEMMA 1. Let $f$ be a phantom map of infinite or finite order from $\Sigma^{k} L(I, J)$ to $S^{k+3}$ for $k \geq 0$. Then, there exits a codiagonal map:

$$
\Delta: C(f) \rightarrow C(f) \vee C(f) .
$$

Proof. Since $j f: L(I, J) \rightarrow S^{3} \rightarrow C(f)$ is zero homotopic, we have $j_{*}(f)=0$ where $j_{*}$ is given by the following diagram:

$$
j_{*}: \operatorname{Ph}\left(L(I, J), S^{3}\right) \rightarrow \operatorname{Ph}(L(I, J), C(f)) .
$$

Since $j_{*}(f)$ is zero, the composition $(j \vee j) d f: L(I, J) \rightarrow S^{3} \rightarrow S^{3} \vee S^{3} \rightarrow$ $C(f) \vee C(f)$ is homotopic to the constant map by using the following diagram (cf. Theorem D of [9]):

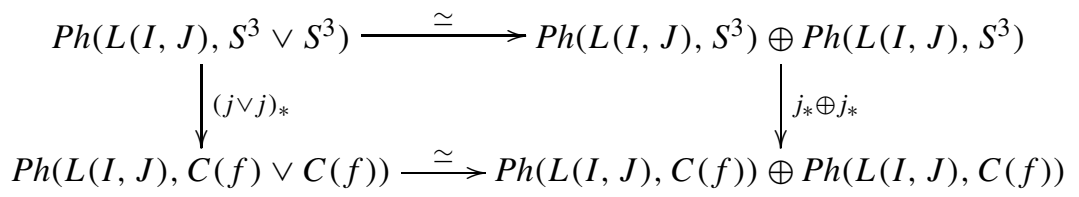

where the left homomorphism is induced by $j \vee j: S^{3} \vee S^{3} \rightarrow C(f) \vee C(f)$.

Consider the following diagram:

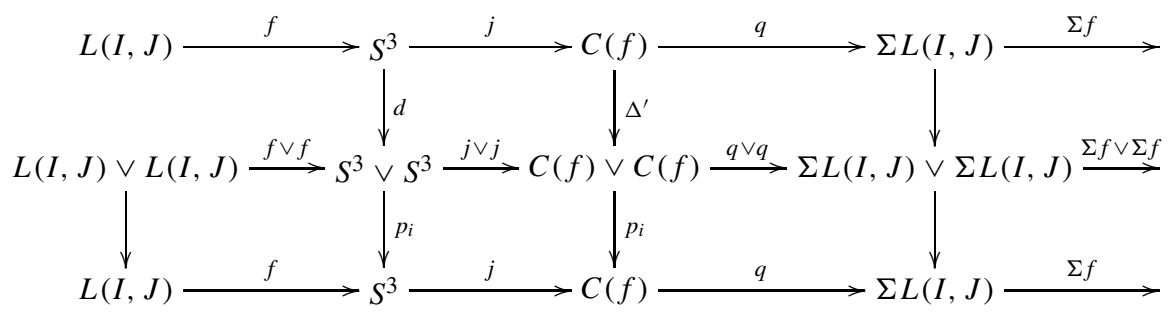

Here, three horizontal sequences are Puppe sequences induced by $f, f \vee f$ and $f$, respectively, $d: S^{3} \rightarrow S^{3} \vee S^{3}$ is a codiagonal map and $p_{i}: X \vee X \rightarrow X$ is a projection for $X=S^{3}, C(f)$ etc, $i=1,2$. We can find out a map $\Delta^{\prime}: C(f) \rightarrow C(f) \vee C(f)$ by the above consideration. If a composed map $p_{i} \Delta^{\prime}$ is not homotopic to id, we have two identity maps $\left(p_{i} \Delta^{\prime}, k_{i}\right) \mu: C(f) \rightarrow C(f)$ by adding maps $k_{i}: \Sigma L(I, J) \rightarrow$ $C(f)(i=1,2)$ where $\mu: C(f) \rightarrow C(f) \vee \Sigma L(I, J)$ is a coaction. Hence we obtain a codiagonal map by adding a map $\left(k_{1} \vee k_{2}\right) v: \Sigma L(I, J) \rightarrow C(f) \vee C(f)$. Now $\Delta$ is given by the following composition:

$\Delta: C(f) \stackrel{\mu}{\longrightarrow} C(f) \vee \Sigma L(I, J) \stackrel{\mu \vee i d}{\longrightarrow} C(f) \vee \Sigma L(I, J) \vee \Sigma L(I, J) \longrightarrow C(f) \vee C(f)$ where the third map is given naturally by $\Delta^{\prime}$ and $k_{i}$. 
We can easily construct a map $\alpha_{m}: C(f) \rightarrow C(m f)$ which is degree $m$ on $S^{3}$ and degree one on cone $L(I, J)$ respectively and $\beta_{n}: C(m n f) \rightarrow C(m f)$ which is degree one on $S^{3}$ and degree $n$ on cone $L(I, J)$ respectively:
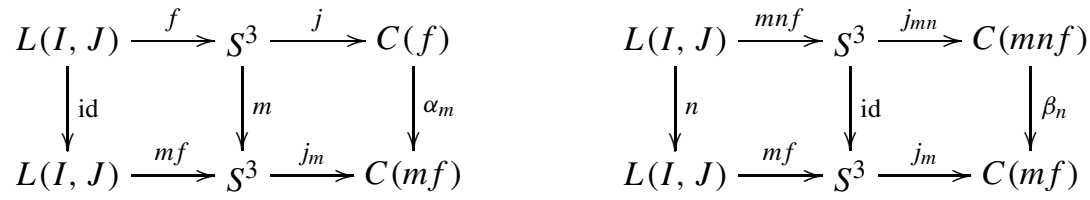

Before giving a homotopy equivalence (3.1), we have to prepare the following. Define a map $\gamma_{k}: C(f) \rightarrow C(f)$ by the following diagram:

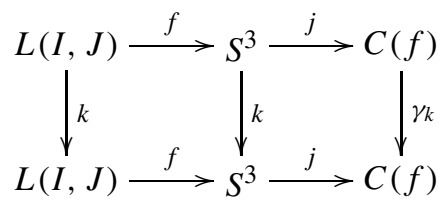

where the first vertical arrow is a map induced by the $\mathrm{H}$-space structure of degree $k$ on the two-dimensional (co)homology group, and the second one is a map of degree $k$. A map $\gamma_{k}^{\prime}: C(f) \rightarrow C(f)$ is defined as follows:

$$
\gamma_{k}^{\prime}: C(f) \rightarrow C(f) \vee \cdots \vee C(f) \rightarrow C(f)
$$

where the first arrow is a $k$-fold codiagonal map and the second one is a folding map. The homomorphisms on homology groups are given as follows:

$$
(\gamma)_{*}(u)=k u, \quad(\gamma)_{*}\left(v_{i}\right)=k^{i} v_{i}, \quad\left(\gamma^{\prime}\right)_{*}(u)=k u, \quad\left(\gamma^{\prime}\right)_{*}\left(v_{i}\right)=k v_{i}
$$

where $u$ and $v_{i}$ are three-dimensional and $(2 i+1)$-dimensional generators of $H_{3}\left(S^{3}\right)$ and $H_{3}(\Sigma L(I, J))$, respectively. By the exactness of the Puppe sequence, we obtain a relation $\left(\gamma_{k}, \delta\right) \mu=\gamma_{k}^{\prime}$ for a map $\delta: \Sigma L(I, J) \rightarrow C(f)$.

We define $\beta_{n}^{\prime}$ and $\beta_{m}^{\prime}$ by adding maps $\delta_{1}$ and $\delta_{2}$ to $\beta_{n}$ and $\beta_{n}$ as above, respectively. The maps $\beta_{n}^{\prime}$ and $\beta_{m}^{\prime}$ satisfy the relations

$$
\left(\beta_{n}^{\prime}\right)_{*}(u)=u, \quad\left(\beta_{n}^{\prime}\right)_{*}\left(v_{i}\right)=n v_{i}, \quad\left(\beta_{m}^{\prime}\right)_{*}(u)=u, \quad\left(\beta_{m}^{\prime}\right)_{*}\left(v_{i}\right)=m v_{i} .
$$

A homotopy equivalence for (3.1) is given by the following composition:

$$
C(f) \vee C(m n f) \rightarrow C(f) \vee C(f) \vee C(m n f) \vee C(m n f) \rightarrow C(m f) \vee C(n f)
$$

where the first map is $\Delta \vee \Delta$ and the second map is given by the following map:

$$
\left(\begin{array}{cccc}
\alpha_{m} & 0 & -t \beta_{n}^{\prime} & 0 \\
0 & \alpha_{n} & 0 & s \beta_{m}^{\prime}
\end{array}\right)
$$


Since it induces an isomorphism on homology groups, it is a homotopy equivalence. From the consideration above, we have the following lemma.

LEMMA 2. Let $f$ be a phantom map of infinite or finite order from $\Sigma^{k} L(I, J)$ to $S^{k+3}$ for $k \geq 0$. Then there exists a homotopy equivalence

$$
C(\text { af }) \vee C(\text { mnaf }) \simeq C(\text { maf }) \vee C(\text { naf })
$$

where $m$ and $n$ are relatively prime integers and $a$ is an integer.

From the lemma, we have the following theorem.

THEOREM 5. Let $f$ be a phantom map of infinite order and $f_{i}=a_{i} f$ for integers $a_{i}$, $(i=1,2, \ldots, n)$. Then there exist phantom maps $g_{i}(i=1,2, \ldots, n)$ of infinite order satisfying the following conditions:

(1) $\bigvee_{i=1}^{n} C\left(f_{i}\right)$ and $\bigvee_{i=1}^{n} C\left(g_{i}\right)$ are homotopy equivalent;

(2) $g_{i}=b_{i} g$ for a phantom map $g$ and integers $b_{i}(i=1,2, \ldots, n)$ and $b_{i+1}$ divided by $b_{i}$ for $i=1,2, \ldots, n-1$.

Although the uniqueness of decomposition fails, it holds for the case of phantom maps of infinite order in the sense of normal form. Moreover, we have the following cancellation theorem.

THEOREM 6. Let $f_{i}$ and $g_{i}(i=1,2, \ldots, n)$ be phantom maps of infinite order and $f_{1}$ and $g_{1}$ homotopic maps. If two wedge sums $\bigvee_{i=1}^{n} C\left(f_{i}\right)$ and $\bigvee_{i=1}^{n} C\left(g_{i}\right)$ are homotopy equivalent, $\bigvee_{j=2}^{n} C\left(f_{j}\right)$ and $\bigvee_{j=2}^{n} C\left(g_{j}\right)$ are homotopy equivalent.

Proof. By Theorem 4, it is sufficient to prove the case for $f_{i}=a_{i} f, g_{i}=b_{i} f$ and $a_{1}=b_{1}$ where $a_{i}, b_{i}$ are integers and $f$ a phantom map. As in the proof of Theorem 3 , we may translate the theorem into a matrix problem. Now we may assume $a_{i+1}$ is divided by $a_{i}(i=2, \ldots, n)$ and also for $b_{i+1}$ and $b_{i}$. We set

(a) $a_{1}=\alpha_{1}, a_{i}=\alpha_{2} \cdots \alpha_{i}(i=2, \ldots, n)$;

(b) $p_{1}=\left(a_{1}, \alpha_{2}\right), p_{i}=\left(a_{1} / p_{1} \cdots p_{i-1}, \alpha_{i+1}\right) i=2, \ldots, n-1, p_{n}=$ $a_{1} / p_{1} \cdots p_{n-1}$.

Here $(a, b)$ means the greatest common divisor of integers $a$ and $b$. By making use of Lemma 2, a diagonal matrix $\operatorname{Diag}\left(a_{1}, a_{2}, \ldots, a_{n}\right)$ can be transformed to $\operatorname{Diag}\left(c_{1}, c_{2}, \ldots, c_{n}\right)$ by elementary unimodular matrices. We obtain the following conditions inductively by the above conditions:

(1) $a_{1}=p_{1} p_{2} \cdots p_{n}$;

(2) $a_{i}=p_{1} p_{2} \cdots p_{i-1} m_{2} \cdots m_{i}, \alpha_{i}=p_{i-1} m_{i}$ for $i>1$;

(3) $\quad c_{i}=p_{1} p_{2} \cdots p_{i} m_{2} \cdots m_{i}$ for $i>1, c_{1}=p_{1}$; 
(4) $\quad m_{i}$ is prime to $p_{i} \cdots p_{n}$ for $i>1$.

Similarly it holds for $\operatorname{Diag}\left(b_{1}, b_{2}, \ldots, b_{n}\right)$ satisfying the following conditions:

(5) $b_{1}=q_{1} q_{2} \cdots q_{n}$

(6) $b_{i}=q_{1} q_{2} \cdots q_{i-1} l_{2} \cdots l_{i}, \beta_{i}=q_{i-1} l_{i}$ for $i>1$;

(7) $d_{i}=q_{1} q_{2} \cdots q_{i} l_{2} \cdots l_{i}$ for $i>1, d_{1}=q_{1}$;

(8) $\quad l_{i}$ is prime to $q_{i} \cdots q_{n}$ for $i>1$.

By the uniqueness of the normal form of a diagonal matrix, we see $c_{i}=d_{i}$ and $p_{i} m_{i}=q_{i} l_{i}$ by $c_{i} / c_{i-1}=d_{i} / d_{i-1}$ for $i=1,2, \ldots, n$. By $c_{n}=d_{n}$ and $a_{1}=b_{1}$, we see $m_{2} \cdots m_{n}=l_{2} \cdots l_{n}$. Hence we have the following equation:

$$
\frac{p_{n} m_{n}}{m_{2} \cdots m_{n}}=\frac{q_{n} l_{n}}{l_{2} \cdots l_{n}}
$$

By cancelling $m_{n}$ and $l_{n}$, we see $p_{n}=q_{n}, m_{n}=l_{n}$ and $m_{2} \cdots m_{n-1}=l_{2} \cdots l_{n-1}$ by the above conditions (4) and (8). By using a descending induction, we see $p_{i}=q_{i}, m_{i}=l_{i}$ and $a_{i}=b_{i}$. Hence we obtain the result.

From the above results, we obtain the cancellation law and the uniqueness of decomposition in the sense of the normal forms for mapping cones of phantom maps of infinite order under the wedge sum operation. Hereafter, we examine the case for phantom maps of finite order. Now we prepare some lemmas of elementary group theory.

LEMMA 3. Let $m, n$ and $a$ be integers such that $m$ and $n$ are relatively prime, and $f$ and $g$ are generators of cyclic groups $Z / m a$ and $Z / n a$, respectively. Then, there exist integers $c$ and $d$ such that $c f+d g$ is a generator of $Z /$ mna and $c$ and $d$ are relatively prime. Here $Z / m a$ and $Z /$ na are considered as subgroups of $Z /$ mna.

Proof. We choose integers $\bar{f}$ and $\bar{g}$ which represent $f$ and $g$, respectively. By assumption, there exist integers $x, y, z, s, t$ and $w$ satisfying $\bar{f} x+$ may $=1$, $\bar{g} z+n a w=1$ and $m s+n t=1$. Set $\bar{F}=n x t \bar{f}+m z s \bar{g}$. This represents a generator of $Z / m n a$ by $n t(x \bar{f}+m a y)+m s(z \bar{g}+n a w)=1$. Moreover, we may assume $\bar{F}=c n \bar{f}+d m \bar{g}=1$ by taking $(n \bar{f}, m \bar{g})=1$ because of a theorem of Dirichlet. Hence $F=c f+d g$ is a generator.

LEMMA 4. Let $f$ and $g$ be generators of $Z / m a$ and $Z / n a$, respectively, and $m$ and $n$ relatively prime. There exist unimodular matrices $P$ and $Q$ satisfying the following:

(1) $P \operatorname{Diag}(f, g)=\operatorname{Diag}(F, G) Q$;

(2) $\operatorname{ord}(F)=$ amn and $\operatorname{ord}(G)=a$. 
Proof. By Lemma 3, we find a generator $F$ of $Z / m n a$ which is represented by $\bar{F}=c n \bar{f}+d m \bar{g}=1$. Set $\bar{G}=m n \bar{g} \bar{f}$ which is a representation of $G$. Since it holds $F=c f+d g$ and $G=m \bar{g} f=n \bar{f} g$, we see $\operatorname{ord}(F)=a m n$ and $\operatorname{ord}(G)=a$ where $\bar{f} n F=f, \bar{g} m F=g$. We obtain the result by defining matrices $P$ and $Q$ as follows:

$$
P=\left(\begin{array}{cc}
1 & d \\
-\bar{g} m & c n \bar{f}
\end{array}\right), \quad Q=\left(\begin{array}{cc}
n \bar{f} & d m \bar{g} \\
-1 & c
\end{array}\right) .
$$

From this lemma, we obtain a normal form of wedge sum decomposition for mapping cones of phantom maps of finite order. From the above equation, we have the following maps:

$$
\begin{aligned}
& \alpha: C(f) \rightarrow C(F), \quad \beta: C(g) \rightarrow C(F) \\
& \gamma: C(f) \rightarrow C(G), \quad \delta: C(g) \rightarrow C(G) .
\end{aligned}
$$

For example, $\alpha$ is defined by the following diagram:

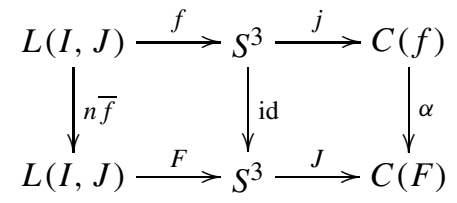

where the first vertical arrow is a map of degree $n \bar{f}$ and the second one is an identity map. The other maps are defined similarly. As in the proof of Lemma 2, we have the following homotopy equivalence:

$$
C(f) \vee C(g) \simeq C(F) \vee C(G)
$$

From this result, we have the following theorem.

THEOREM 7. Let $f_{i}(i=1,2, \ldots, n)$ be phantom maps of finite order. There exist phantom maps $g_{i}(i=1,2, \ldots, n)$ of finite order satisfying the following conditions:

(1) $\bigvee_{i=1}^{n} C\left(f_{i}\right) \simeq \bigvee_{i=1}^{n} C\left(g_{i}\right)$;

(2) $\operatorname{ord}\left(g_{i}\right)$ is divided by ord $\left(g_{i+1}\right)$.

We study Problem 2. That is, does a cancellation law hold for phantom maps of finite order? We can easily give counterexamples.

Example 1. Let $f$ be a phantom map of order $m n$ and integers $a, n$ satisfy $a x+m n y=$ 1 for integers $x, y$. Set $g=m f$. If $g$ satisfies $a g=g$, the following equation holds:

$$
\left(\begin{array}{cc}
a f & 0 \\
0 & g
\end{array}\right)\left(\begin{array}{cc}
x & m \\
-n y & a
\end{array}\right)=\left(\begin{array}{cc}
x a & 1 \\
-m n y & 1
\end{array}\right)\left(\begin{array}{ll}
f & 0 \\
0 & g
\end{array}\right) .
$$


Hence, we see that $C(a f) \vee C(g) \simeq C(f) \vee C(g)$. Here ord $(f)=\operatorname{ord}(a f)=m n$, $\operatorname{ord}(g)=n$. This shows that a cancellation law does not hold.

Example 2. Let $f$ be phantom maps of order $m n$ and $g=m f$. Then, we choose integers $a, s$ such that $a s+m n t=1$ and $s x+n y=1$. We have the following equation:

$$
\left(\begin{array}{cc}
a & -t n \\
m & s
\end{array}\right)\left(\begin{array}{ll}
f & 0 \\
0 & g
\end{array}\right)=\left(\begin{array}{cc}
a f & 0 \\
0 & s g
\end{array}\right)\left(\begin{array}{ll}
1 & 0 \\
x & 1
\end{array}\right) .
$$

From this, we see that $C(f) \vee C(g) \simeq C(a f) \vee C(s g)$. Generally, $C(f)$ and $C(a f)$ are not homotopy equivalent and also for $C(g)$ and $C(s g)$.

Although a cancellation law dose not hold for the case of phantom maps of finite order, the uniqueness of decomposition holds in some sense. That is, we have the following lemma.

LEMMA 5. Let $f_{i}, g_{i}(i=1,2, \ldots, n)$ be phantom maps of finite order such that $\operatorname{ord}\left(f_{i}\right)$ is divided by $\operatorname{ord}\left(f_{i+1}\right)$ and also for $g_{i}^{\prime} s$. If there exist unimodular matrices $A, B$ such that

$$
A \operatorname{Diag}\left(f_{1}, f_{2}, \ldots, f_{n}\right)=\operatorname{Diag}\left(g_{1}, g_{2}, \ldots, g_{n}\right) B
$$

then $\operatorname{ord}\left(f_{i}\right)=\operatorname{ord}\left(g_{i}\right)$ for $i=1,2, \ldots, n$.

Proof. Note that $\operatorname{ord}(f)$ is equal to 1 for a trivial phantom map $f$. We set $\operatorname{ord}\left(f_{i}\right)=$ $m_{i} \cdots m_{n}, \operatorname{ord}\left(g_{i}\right)=l_{i} \cdots i_{n}$ for $i=1,2, \ldots, n$. From equation $(*)$, we have

(a)

$$
a_{i j} f_{j}=g_{i} b_{i j} \quad(i, j=1,2, \ldots, n) .
$$

Since $\operatorname{det} A=+1,-1$,

(b)

$$
\begin{aligned}
f_{k} & = \pm(\operatorname{det} A) f_{k} \\
& = \pm \sum a_{\sigma(1) 1} \cdots a_{\sigma(k) k} \cdots a_{\sigma(n) n} f_{k} \\
& = \pm \sum a_{\sigma(1) 1} \cdots a_{\sigma(k-1) k-1} b_{\sigma(k) k} a_{\sigma(k+1) k+1} \cdots a_{\sigma(n) n} g_{\sigma(k)} \\
& =\text { Sum of type } 1+\text { Sum of type } 2 .
\end{aligned}
$$

Here, type 1 is the summation satisfying $\sigma(k)$ greater than $k-1$ and type 2 is that with $\sigma(k)$ smaller than $k$. Clearly the order of type 1 is equal to the factor of $\operatorname{ord}\left(g_{k}\right)$. We prove the theorem by induction on $k$. Since for the case $k=1$, there is no term of type $2, \operatorname{ord}\left(f_{1}\right)$ divides ord $\left(g_{1}\right)$, that is, $m_{1} m_{2} \cdots m_{n}$ divides $l_{1} l_{2} \cdots l_{n}$. Conversely, ord $\left(g_{1}\right)$ 
divides ord $\left(f_{1}\right)$, hence $\operatorname{ord}\left(f_{1}\right)=\operatorname{ord}\left(g_{1}\right)$. Now we assume $\operatorname{ord}\left(f_{i}\right)=\operatorname{ord}\left(g_{i}\right)$ for $i<k$. From (a) and the hypothesis of induction, $a_{\sigma(i) i}$ is divided by $l_{i} l_{i+1} \cdots l_{\sigma(i)-1}$ for the case when $\sigma(i)$ is greater than $i, i=1,2, \ldots, k-1$. Define $N_{\sigma}$ for a permutation $\sigma$ as follows:

$$
N_{\sigma}=\prod_{\substack{\sigma(i)>i, i=1, \ldots, k-1}}\left(l_{i} l_{i+1} \cdots l_{\sigma(i)-1}\right) .
$$

Since $N_{\sigma}$ is divided by $l_{\sigma(k)} \cdots l_{k-1}$ for the case of $\sigma(k)<k$ in type $2, N_{\sigma} g_{\sigma(k)}$ has an order $l_{k} l_{k+1} \cdots l_{n}$ at most. Since $a_{\sigma(1) 1} a_{\sigma(2) 2} \cdots a_{\sigma(k-1) k-1}$ is divided by $N_{\sigma}$, type 2 has an order $l_{k} l_{k+1} \cdots l_{n}$ at most. Since the order of type 1 divides $l_{k} l_{k+1} \cdots l_{n}$, we see that the order of $f_{k}$ divides $l_{k} l_{k+1} \cdots l_{n}$. Conversely the order of $g_{k}$ divides $m_{k} m_{k+1} \cdots m_{n}$. Hence ord $\left(f_{i}\right)=\operatorname{ord}\left(g_{i}\right)$.

From the above lemma and the proof as in Theorem 3, we obtain the following theorem.

THEOREM 8. Let $f_{i}(i=1,2, \ldots, n)$ be phantom maps of finite order such that $\operatorname{ord}\left(f_{i}\right)$ is divided by ord $\left(f_{i+1}\right)$ and also for $g_{i}$ 's. If $\bigvee_{i=1}^{n} C\left(f_{i}\right)$ and $\bigvee_{i=1}^{n} C\left(g_{i}\right)$ are homotopy equivalent, it holds that $\operatorname{ord}\left(f_{i}\right)=\operatorname{ord}\left(g_{i}\right)$ for $i=1,2, \ldots, n$.

Proof. Let $\phi: \bigvee_{j=1}^{n} C\left(f_{i}\right) \rightarrow \bigvee_{j=1}^{n} C\left(g_{i}\right)$ be a homotopy equivalence. As in the proof of Theorem 3, we consider

$$
\begin{aligned}
H_{3}(\phi) & =\left(A_{i j}\right) \quad(i, j=1,2) \\
A_{i j}: H_{3}\left(X_{j}\right) & \rightarrow H_{3}\left(X_{i}\right),
\end{aligned}
$$

where $X_{i}$ is $n S^{3}$ and $n \Sigma L(I, J)$ for $i=1$ and 2, respectively. We see that the small matrix $A_{12}$ is zero and the $i$ th column of $A_{21}$ is a multiple of ord $\left(f_{i}\right)$ by the same method of Section 3 in [6]. By Proposition 3.5 of [6], we can also construct a map of homotopy equivalence:

$$
\psi: \bigvee_{j=1}^{n} C\left(f_{i}\right) \rightarrow \bigvee_{j=1}^{n} C\left(f_{i}\right)
$$

which is represented by the following matrix:

$$
\left(\begin{array}{cc}
I & 0 \\
-A_{22}^{-1} A_{21} & I
\end{array}\right) \text {. }
$$

The composition map $\phi \psi$ gives a homotopy equivalence:

$$
\bigvee_{i=1}^{n} S^{3} \rightarrow \bigvee_{i=1}^{n} S^{3}
$$


As in the proof of Theorem 3, we obtain the equation:

$$
A_{11} \operatorname{Diag}\left(f_{1}, f_{2}, \ldots, f_{n}\right)=\operatorname{Diag}\left(g_{1}, g_{2}, \ldots, g_{n}\right) A_{22} .
$$

Hence we have the result by Lemma 5 .

Acknowledgement. The author would like to thank a referee for his useful comments.

\title{
REFERENCES
}

[1] P. Freyd. Stable homotopy 1. Proc. Conf. Categorical Algebra (La Jolla, 1965). Springer, New York, 1966.

[2] P. Freyd. Stable homotopy 2. Amer. Math. Soc. Proc. Symp. Pure Math. 18 (1970), 161-183.

[3] C. A. McGibbon. The Mislin genus of a space. Centre de Recherches Mathematiques, CRM Proc. Lecture Note. 6 (1994), 75-102.

[4] G. Mislin. Cancellation properties of H-spaces. Comment. Math. Helv. 49 (1974), 195-200.

[5] Y. Shitanda. Uncountably many loop spaces of the same $n$-type for all $n, 1,2$. Yokohama Math. J. 41 (1993), 17-24; Yokohama Math. J. 41 (1994), 85-93.

[6] Y. Shitanda. Cancellation and non cancellation phenomena for infinite complexes. Publ. RIMS, Kyoto Univ. 32 (1996), 383-399.

[7] Y. Shitanda. Spaces of the same clone type. J. Math. Soc. Japan 48(4) (1996), 705-714.

[8] C. Wilkerson. Genus and cancellation. Topology 14 (1975), 29-36.

[9] A. Zabrodsky. On phantom maps and a theorem of H. Miller. Israel J. Math. 58(2) (1987), 129-143.

\author{
Yoshimi Shitanda \\ Meiji University \\ Izumi Campus \\ Eifuku 1-9-1, Suginami-ku \\ Tokyo 168, Japan \\ (E-mail: shitanda@isc.meiji.ac.jp)
}

